\title{
La relación escuela - comunidad: un análisis desde la teoría de sistemas a nueve experiencias de América Latina
}

José Darío Herrera ${ }^{1}$

Recibido: 13-03-2016

Aceptado: 05-05-2016

\section{RESUMEN}

Este artículo busca contribuir a la comprensión de la relación escuelacomunidad. Se examinaron nueve proyectos implementados en las dos últimas décadas en Latinoamérica cuyo propósito constituía articular experiencias de desarrollo de base con el trabajo escolar. El estudio concluyó que existen iniciativas y acciones en las que el campo del desarrollo de base puede aportar al mejoramiento de la educación; es importante diferenciar entre impacto directo o indirecto al hablar de las incidencias que desde un campo se puede hacer sobre el otro; las experiencias trabajan directa o indirectamente sobre desarrollo de base y educación al situarse frente a una problemática que los articula y que hace parte de las necesidades de los territorios; se percibe una tendencia macro: el desarrollo de base al servicio de la educación, más que la educación en función del desarrollo de base y el aporte que los modelos pedagógicos pueden hacer a la cualificación del capital social.

Palabras clave: Desarrollo de base, educación, comunidad.

1. Doctor en Filosofía de la Universidad Nacional de Colombia. Autor de Pensar la educación, hacer investigación (2013), La comprensión de lo social. Horizonte hermenéutico de las ciencias sociales (2010) y Comanche, Comandante del Cartucho (1995). Línea de investigación: Gestión educativa y políticas públicas. Universidad de los Andes. Facultad de Educación. Profesor Asociado. Calle 18 A N ํㅣ-19 Este.

Correo: jd.herrera@uniandes.edu.co

Teléfono: 3394999 ext. 3077 


\title{
School Community Relations: an AnalysisFrom the theory of Nine Systems Experiences from Latin America
}

\begin{abstract}
This article seeks to contribute to the understanding of the relationship school community. There were examined nine experiences implemented in last two decades in Latin America which intention constituted to articulate base development experiences with the school work. It was concluded that: there are initiatives and actions in which the field of base development can contribute to the improvement of education; It is important to distinguish between direct or indirect impact to speak of incidents that can be made from a field on the other; the experiences working directly or indirectly on base development and education to be facing a problem that articulates them and makes part of the needs of the territories; a macro trend is perceived: the base development at the service of education more than the education according to the base development and the contribution that the pedagogical models can be made to the qualification of social capital.
\end{abstract}

Keywords: Base development, education, community.

\section{RESUMO}

Este artigo busca contribuir com a compreensão da relação entre a escola e a comunidade. Para isso, foram examinadas nove experiências implementadas nas últimas duas décadas na América Latina cujo propósito constituía articular experiências de desenvolvimento de base com o trabalho escolar. O estudo concluiu que: existem iniciativas e ações nas que o campo do desenvolvimento de base pode contribuir ao melhoramento da educação; é importante diferenciar entre impacto direto ou indireto ao falar das incidências que desde um campo pode fazer sobre o outro; as experiências trabalham direta ou indiretamente sobre desenvolvimento de base e educação ao se situar frente a uma problemática que os articula e que faz parte das necessidades dos territórios; se percebe uma tendência macro: o desenvolvimento 
de base ao serviço da educação mais que a educação em função do desenvolvimento de base e a contribuição que os modelos pedagógicos podem dar à qualificação do capital social.

Palavras-chave: Desenvolvimento de base, educação, comunidade.

\section{Introducción}

De acuerdo con el Informe de la Situación Educativa de América Latina y el Caribe (UNESCO, 2013), la región Latinoamericana "ha presentado un desarrollo importante en las últimas dos décadas, el crecimiento económico ha sido sostenido..." (p. 124). Hubo signos de mejoría en lo que respecta a las condiciones de vida de la población y un significativo progreso en cuanto a la alfabetización y acceso a la educación. Sin embargo, de acuerdo con el informe, "la desigualdad social es una característica relevante en la región y representa el principal desafío a hacer frente" (UNESCO, 2013, p. 57), situación que afecta la consecución de las metas propuestas por la UNESCO para el siglo XXI. Problemas como la pobreza y el lento desarrollo social de varias comunidades afectan la calidad de la educación recibida por los sectores más pobres de la sociedad. Asimismo, las diferencias en la calidad de los procesos educativos están ligadas a razones étnicas, nivel socioeconómico, zona de residencia y de género, (UNESCO, 2013, p. 57) aspectos que sobrepasan los objetivos de las políticas educativas.

En este contexto y en parte como consecuencia de la incapacidad de la política pública para enfrentar las desigualdades estructurales, desde los años go la responsabilidad por la calidad de la educación ha sido compartida entre el estado y otros actores sociales (Gajardo y Puryear, 2003). Padres, organizaciones comunitarias y organizaciones no gubernamentales, han jugado un papel importante en dicha tarea.

A partir de una encuesta aplicada por RedeAmérica a las fundaciones y corporaciones sobre sus proyectos con escuelas y comunidades, se seleccionaron nueve de sus experiencias de trabajo de las últimas dos décadas en Latinoamérica para un estudio de caso múltiple. Este artículo pretende realizar una contribución para entender la relación Escuela- 
Comunidad. Se enfatiza en los problemas que vinculan a las comunidades con la educación, problemas que al decir de Niklas Luhmann (1998) pueden ser entendidos como "equivalentes funcionales" en la construcción del tejido social.

El artículo se estructura en cuatro apartados:

I. Tejido Social: Una Posibilidad para pensar las relaciones del Desarrollo de Base y la Educación.

II. Escuela y Comunidad

III. Tipos de experiencias que articulan Desarrollo de Base y Educación: Nueve casos en América Latina.

IV. Problemáticas Vinculantes

\section{Tejido social: una posibilidad para pensar las relaciones del desarrollo de base y la educación}

La noción de tejido social llama la atención respecto de la interrelación existente entre los distintos ámbitos de la vida social y ha sido empleada en nuestro contexto sobre todo por quienes se encargan de estudiar los efectos negativos que fenómenos como la violencia, la corrupción, la pobreza o el narcotráfico tienen sobre el conjunto de la vida social; se habla entonces de la reparación del tejido social. La metáfora, como puede verse, resalta la interconexión existente entre diversos aspectos de la vida comunitaria e invita a comprender la realidad social más como un entramado de relaciones que como la sumatoria de unidades aisladas unas de otras. Teóricamente, la noción de tejido social se soporta en la teoría de los sistemas sociales autorreferentes muy conocida hoy gracias a los trabajos del alemán Niklas Luhmann (1998) a principios de los 80.

La intención de Luhmann es ofrecer "una teoría policéntrica y por consiguiente policontextual, en un mundo y una sociedad concebidos acéntricamente" (Luhmann, 1998, p. 11) para lo cual desarrolla un método de análisis social que resalta las interconexiones y superposiciones entre variados aspectos de la vida social y que él denomina el "método funcional". Este método se propone frente a la clásica idea de entender la ciencia como "conocimiento de los fenómenos por sus causas". El propósito de Luhmann (1998) es apartarse de la idea de que cada fenómeno específico, por ejemplo, la deserción escolar, deba ser 
explicado a partir de causas igualmente específicas como la precaria situación económica de las familias, para el caso.

En oposición a ello, este pensador alemán, plantea la idea de "equivalentes funcionales" que consiste en lo siguiente: una vez establecido un problema social, se determinan aquellos hechos sociales que aunque en apariencia sean distintos pueden ser tratados como posibles o deseables explicaciones. Es decir, si para el caso de la deserción escolar, se quiere establecer los posibles o deseables equivalentes funcionales, lo primero que se debe hacer es tomar la "deserción escolar" no como un "efecto" atribuible a una o varias "causas" sino como un "problema" que puede ser explicado y transformado a partir de múltiples opciones; todas ellas equivalentes en la medida en que están en función de resolver o explicar el problema.

La novedad más importante en esta propuesta consiste en superar el determinismo que hace ver los fenómenos sociales como efectos de causas específicas para entrar a comprenderlos como problemas que pueden ser explicados y transformados en la medida en que muchos otros hechos sociales, a veces, sin conexión clara desde el pensamiento causal, puedan ser asumidos en función de su solución o explicación. Para el caso del ejemplo que se ha venido tomando, la novedad se podría ilustrar así: mientras el pensamiento causal determina la causa o las causas que hacen que se presente la deserción escolar e insiste en modificar esa situación para evitar esa consecuencia, el pensamiento "sistémico" hace notar que cualquier otro aspecto de la vida social (la participación en los grupos pastorales de la iglesia local, por ejemplo) sin conexión causal aparente con el fenómeno en cuestión, puede entrar a formar parte de la solución del problema. Se pasa pues de considerar la realidad social como una gama de fenómenos determinados por causas a considerarla como un sistema dinámico que se articula de maneras a veces sorprendentes pero siempre en constante transformación. En consecuencia, las relaciones causales pasan a ser pensadas como relaciones de posibilidad.

Para el caso de interés -la superposición entre los campos del desarrollo de base ${ }^{2}$ y la educación- es decir, no se trata de establecer relaciones

2. de acuerdo con RedEAmérica (2007), el desarrollo de base es "la capacidad colectiva de las organizaciones comunitarias para poner en movimiento por sí mismas los recursos humanos, físicos y económicos disponibles en ellas y en su entorno, definir sus necesidades, identificar las alternativas de acción más viables para la superación de sus problemas, e identificar, formular, ejecutar y evaluar planes, programas y proyectos de desarrollo. También consiste en el incremento y cualificación de la interacción de las organizaciones entre sí, y de éstas con los actores públicos y privados, de manera que se consoliden entornos favorables a sus iniciativas y a su participación permanente en los asuntos públicos". (p.10) 
causales entre los dos sino más bien, y en un sentido muy cercano a los planteamientos de Luhmann (1998), de pensar relaciones de posibilidad. Los fenómenos sociales al ser considerados como problemas restituyen la generalidad que el esquema cerrado de la causalidad no dejaba pensar. La noción de tejido social resalta precisamente esto: la interconexión de todo con todo. Y al hacerlo, orienta nuestra mirada, no hacia la particularidad de cada cosa, sino hacia el sistema que lo explica y que permite pensar en su transformación. Se resaltan las conexiones más que lo conectado, es más, las "partes" del sistema sólo son comprensibles a partir de las conexiones existentes y posibles. A esto se refiere el adjetivo "autorreferentes" cuando se habla de sistemas sociales. Cada aspecto del sistema es comprensible a partir de las relaciones que el mismo sistema establece en su interior. La escuela, por ejemplo, sólo puede ser comprendida y transformada por las múltiples funciones que toma dentro del sistema social. Su comprensión está dada por el lugar que ocupa dentro de las complejas y variadas relaciones que el sistema establece más que por la definición de unos objetivos o propósitos hechos de espaldas a los contextos en los que funciona.

Es de interés destacar la noción de problema que propone la teoría de Luhmann (1998), pues, como se verá más adelante, esta noción juega un papel central en las experiencias que vienen pensando las posibles relaciones entre desarrollo de base y educación. La noción de problema dice Luhmann (1964) es un "criterio de referencia abstracto" que permite vincular muchos aspectos de la vida social como "equivalentes funcionales", es decir, que permite asociar los más diversos aspectos de la realidad social en torno a algo. Es justo aquí que cobra tanta importancia la noción de problema. Ya se ha dicho que esta noción restituye el carácter de generalidad que tienen todos los fenómenos sociales, pues atañen a todo el sistema; se debe decir, ahora, que el concepto de problema sirve como criterio para ordenar la complejidad de lo social.

Hacer visible el tejido social supone entonces emplear este tipo de criterios abstractos a los que se refiere Luhmann (1998) pero la selección de estos criterios no puede hacerse de espaldas al tejido social que se está analizando. Esto significa que no cualquier criterio puede ser utilizado y que es necesario prestar atención a aquellos temas que presentan alguna relevancia para los distintos agentes del tejido social. Si se establece por ejemplo la deserción escolar como un problema, se hace suponiendo, ya que este tema es interesante para aquellos que habitan el contexto que se busca estudiar. 
Una vez establecido el problema "deserción escolar", el paso a seguir consiste en establecer las posibles relaciones de este problema con los múltiples aspectos de la realidad social. Aparecerán entonces en un diagrama o en un mapa el criterio ordenador (deserción escolar) y en torno a él distintas líneas que expresan a su vez las relaciones existentes o posibles entre ese problema y las demás partes del sistema. Si se ubica otro criterio, por ejemplo, el maltrato infantil, los equivalentes funcionales expresados en el primer diagrama tomarán otro sentido en función del nuevo problema. Incluso puede resultar que aparezcan otros ámbitos de la realidad social que no aparecían en el primer diagrama, la inspección de policía para poner un caso. La complejidad entonces de un sistema va apareciendo en la medida en que se determinen problemas y se tracen relaciones. Si se superponen varios diagramas de este tipo, la imagen que resulta al final se parece a un tejido.

Expuesta así, la noción de tejido social resulta más útil para comprender las relaciones existentes y posibles entre el desarrollo de base y educación. No se trata de establecer relaciones de causalidad, es decir, de saber si tal aspecto del desarrollo de base causa tal efecto en la educación o de si tal aspecto de la educación causa tal efecto en el desarrollo de base; se trata de ver ambos campos como partes de un sistema en el que cada parte puede ser comprendida solo por referencia a las otras. Asimismo, las posibilidades de transformación tienen que ver justamente con el poder establecer nuevas relaciones. Es precisamente esto lo que buscan las experiencias que intentan articular lo que sucede en la escuela con lo que sucede en las organizaciones de base.

Antes de entrar en las experiencias, se mencionará brevemente el campo temático en el que vale la pena su análisis.

\section{Escuela y comunidad}

Los estudios sobre la relación entre la escuela y la comunidad (Trilla, 1995, 1999; Merino, 2005, 2008; Fernández, 2009) se clasifican en dos modelos: Escuela Clausurada y Escuela Abierta. En la primera, la escuela tiende a cerrarse sobre sí misma como un espacio ajeno al contexto que toma de él sólo aquello que necesita y funciona ignorando su entorno sociocultural. La escuela clausurada se cierra sobre sus propias lógicas de trabajo curricular y crea una cultura escolar completamente aislada 
de la cultura social (Fernández, 2009). En el segundo modelo, la escuela se involucra con el entorno tanto como es posible (Trilla, 1995, 1999), incorpora el conocimiento de la comunidad y participa activamente dentro de ella transformando sus dinámicas (Trilla, 1999). La expresión "la escuela en la comunidad y la comunidad en la escuela" expresa el reto actual de desarrollar una escuela abierta al entorno, equitativa e inclusiva, frente al anacronismo que significa el modelo de la mal denominada "escuela tradicional" de corte racionalista y clausurada sobre sí misma (Fernández, 2009). Ahora bien, a pesar de que el interés por superar la brecha entre las comunidades y las instituciones educativas, la disociación entre la escuela y la vida cotidiana de los estudiantes continúa siendo un problema central para la educación (García, 2005; Parrilla, 2002; Booth, 2000).

En este sentido, los estudios sobre las relaciones entre la comunidad y la escuela resaltan la importancia de aumentar las oportunidades de aprendizaje fuera de la escuela, e indican la necesidad de vincular los sistemas formales y no formales de educación para mejorar los procesos de aprendizaje de los estudiantes (Torres, 2001, Durston, 1995, Herrera, 2005). Si bien, una escuela abierta a su contexto posibilita mejores aprendizajes, el modelo de escuela abierta también reta a que las escuelas se hagan cargo de muchas necesidades de sus contextos, en palabras de Fernández (2009) "el desafío de cambio apuntado requiere que la escuela se abra a la sociedad haciéndose cada vez más permeable a la misma en la doble dirección de permeabilidad hacia fuera (escuela hacia el entorno) y de permeabilidad hacia dentro (entorno hacia la escuela), puesto que es en la sociedad donde el hombre vive y en donde se generan sus necesidades socio-educativas" (p. 34).

Dicho así, la escuela se convierte en una instancia de redefinición y reconstrucción social de las culturas locales. En la investigación elaborada con nueve de las experiencias de la organización RedEAmérica de las últimas dos décadas, se identificaron tres tipos de trabajo y cuatro problemas vinculantes. A continuación se presentan esos resultados. 


\section{III.Tipos de experiencias que articulan de sarrollo de base y educación: nueve casos en América Latina}

Las organizaciones que participaron del estudio fueron seleccionadas de entre las más de 140 experiencias de las últimas dos décadas en Latinoamérica, vinculadas a RedEAmérica de acuerdo con los siguientes criterios: la articulación tanto con actores locales como con los planes locales de educación, la participación de organizaciones de base y las metas establecidas. Por lo tanto, estas nueve experiencias incluyen la participación de organizaciones de base en el desarrollo educativo de sus contextos. En la siguiente tabla se especifican el país, el programa, y la organización, miembro de RedEAmérica, a cargo de cada una de las experiencias seleccionadas.

Tabla 1. Nueve experiencias en Latinoamérica.

\begin{tabular}{|l|l|l|}
\hline ORGANIZACIÓN & PAÍs & PROGRAMA \\
\hline Arcor & Argentina & Oportunidades educativas y comunitarias \\
\hline Minetti & Argentina & Juntos por la educación \\
\hline Hedging Griffo & Brasil & Programa CRESCER \\
\hline Camargo Correa & Brasil & Todos por la educación \\
\hline Smurfit & Colombia & Instituto técnico ITAF \\
\hline Fundación Corona & Colombia & Fondo Focus \\
\hline $\begin{array}{l}\text { Empresarios por } \\
\text { la educación }\end{array}$ & Colombia & Modelos escolares para la equidad \\
\hline $\begin{array}{l}\text { Fundación } \\
\text { Falconbridge }\end{array}$ & $\begin{array}{l}\text { República } \\
\text { Dominicana }\end{array}$ & Programa de apadrinamiento de escuelas \\
\hline Wong & Ecuador & Sistema de aprendizaje tutorial (SAT) \\
\hline
\end{tabular}

Fuente. Propia

Las experiencias documentadas adoptan distintos enfoques de acción en torno al desarrollo de base y educación, y definen de diferentes formas su participación en los programas y proyectos que dinamizan, de acuerdo con las problemáticas sobre las cuáles buscan incidir y los procesos que buscan consolidar. Sin embargo, se encuentra que las experiencias pueden responder a tres formas predominantes de trabajo que aunque no se excluyen entre sí, plantean en su implementación formas específicas de trabajo en el campo de desarrollo de base y la educación: 
1. Experiencias que tienen por objetivo que las comunidades mismas definan las problemáticas educativas presentes en su territorio y que, a partir de ellas, generen prácticas de desarrollo de base ligadas a la participación en escenarios de construcción de políticas públicas.

2. Experiencias que tienen por objetivo apoyar el trabajo pedagógico que realizan las instituciones escolares en las comunidades.

3. Experiencias que tienen por objetivo incidir en la apertura y cualificación de ambientes educativos en las comunidades.

A continuación se describirá de una manera más detallada estos tres tipos de experiencias en el campo del desarrollo de base y la educación.

\section{Primer tipo de experiencia: la participación de la comunidad en la construcción de una agenda de política pública para la incidencia en las problemáticas educativas del territorio}

En este tipo de trabajo se pueden ubicar las siguientes experiencias:

Oportunidades Educativas Comunitarias.

Fundación Arcor (Argentina), Juntos por la Educación, Fundación Minetti (Argentina), Fondo FOCUS, Fundación Corona, (Colombia) y Programa Todos por la Educación, Instituto Camargo Correa (Brasil).

Estas experiencias se caracterizan, en su conjunto, por apuntar a la construcción de espacios de concertación y de articulación de esfuerzos transectoriales para solucionar las problemáticas educativas del territorio, las cuales no se entienden solamente como problemáticas ligadas a la institución escolar, sino que se definen desde un contexto más amplio relacionado con el desarrollo de los niños y niñas, y la constitución del ámbito comunitario como un espacio social para la construcción y protección de la infancia. Por esta razón, las experiencias aquí relacionadas se caracterizan porque promueven que las comunidades realicen sus propios diagnósticos sobre la situación educativa local a partir de un problema central: las condiciones de vida de los niños y las niñas. De este modo, se construye una mirada sobre lo educativo que no sólo considera al niño y a la niña en relación con el espacio escolar, sino que se pregunta por las formas de socialización propias del territorio a partir de las cuales se construyen las identidades y se forjan los aprendizajes en la infancia.

Se trata, entonces, de una perspectiva de trabajo que se enfoca en que las comunidades generen y gestionen diversas estrategias o dispositivos 
de socialización que incidan en la calidad de vida de los niños y las niñas. Por ello es esencial, en este tipo de intervención, la construcción por parte de las comunidades y la capacidad para trabajar con distintas organizaciones sociales, públicas y privadas, de tal manera que las necesidades educativas del territorio formen parte de una agenda de política pública, que articule los esfuerzos y recursos de los diversos actores sociales.

En este sentido, las experiencias pueden adelantar, como en el caso del programa Todos por la Educación del Instituto Camargo Correa, acciones de formación al profesorado con el fin de incidir a través de ellos en el mejoramiento de las condiciones de vida de los niños y niñas, o acciones de trabajo pedagógico con jóvenes en un contexto extraescolar. De la misma manera, en el caso del programa Oportunidades Educativas Comunitarias, el programa Juntos por la Educación y el programa Fondo FOCUS de la Fundación Corona crean estrategias de articulación a nivel local, para generar acciones sustentables frente a las necesidades de los niños, niñas y jóvenes y crear condiciones para elevar el nivel de vida de las comunidades desde proyectos socioeducativos (Ancor yMinetti, , 2006; (EPYD, 2001; Corona, 2004).

Así pues, el acompañamiento de los procesos de desarrollo de base se caracteriza por tener procesos de identificación y mapeado de las organizaciones sociales presentes en el territorio y convocatoria a los actores sociales y procesos de formación en temas relacionados con la autogestión de las organizaciones de base: planeación estratégica, formulación, implementación y evaluación de proyectos, manejos de presupuestos, consecución de recursos. Asimismo, por contar con el apoyo a las comunidades en la identificación de las problemáticas ligadas a un tema transversal al desarrollo de base, como la infancia. De la misma manera, con procesos de formalización de espacios locales para la incidencia en políticas públicas y promoción de alianzas en torno a temas transectoriales y procesos de investigación, evaluación, sistematización y difusión de las experiencias de desarrollo de base.

\section{Segundo tipo de experiencias: el fortalecimiento de las instituciones escolares}

En este tipo de trabajo se pueden ubicar las siguientes experiencias: proyecto Modelos Escolares para la Equidad, Fundación Empresarios por la Educación (Colombia), Institutos Técnicos Agropecuarios y Forestales, 
Fundación Smurfit. (Colombia) y Apadrinamiento a Escuelas, Fundación Falconbridge. (República Dominicana).

A diferencia del primer tipo de experiencias, este segundo tipo focaliza su trabajo en la institución escolar. Esto es, que desde las organizaciones socialesseapoyaalaescuela enlaresolución delasproblemáticaseducativas que le son inherentes (fracaso escolar, deserción, bajos aprendizajes), o se apunta a fortalecer la escuela y los procesos pedagógicos escolares para convertirla en un actor clave para el desarrollo de las comunidades. En contraste con las experiencias ubicadas en el primer tipo, que focalizan su trabajo en el mejoramiento de las condiciones de vida de la infancia, el punto focal de estas experiencias son los aprendizajes escolares.

Por esta razón estas experiencias buscan empoderar a las instituciones educativas a través de la articulación de dos formas de gestión: la gestión pedagógica y la gestión del contexto. La gestión pedagógica implica la implementación de modelos pedagógicos y de estructuras curriculares que posibilitan el diálogo de las prácticas escolares con el contexto sociocultural de los estudiantes. La gestión de contexto, por su parte, articula la oferta cultural de las comunidades a los procesos pedagógicos escolares.

Así pues, la gestión pedagógica se realiza de dos formas, cada una de las cuales hace énfasis en algún aspecto de la institución. O bien se insiste en las dinámicas del aula de clase o se enfatiza en crear las condiciones institucionales que mejoren las dinámicas de aula. En el caso de los ITAF, tiene que ver con el diseño e implementación de propuestas curriculares y planes de estudio, el desarrollo de materiales didácticos, procesos de formación del profesorado, de acompañamiento in situ a proyectos pedagógicos y el mejoramiento de los espacios físicos de la escuela. Se trata, entonces, de que las aulas incorporen la vida y los saberes de las comunidades como factores de aprendizaje de los niños, niñas y jóvenes. La articulación de los saberes escolares con los saberes de las comunidades se convierte así en un elemento que favorece la retención de los niños y niñas en el sistema escolar y que promueve, al mismo tiempo la participación de los niños, niñas y jóvenes en las comunidades (Smurfitkappa, 2014).

En el caso del proyecto MEPE, los actores escolares participan en un proceso de caracterización de la realidad de la institución escolar, con el fin de levantar una línea de base y de diseñar proyectos tendientes 
a transformar los procesos pedagógicos e institucionales. El modelo diseñado por el proyecto entra en diálogo con el contexto y con las prácticas y saberes que históricamente se encuentran presentes en la institución escolar y se hace operativo a través de los actores escolares mismos: directivos, profesores, estudiantes, padres de familia. (Empresarios por la Educación, 2010). En el caso del programa de Apadrinamiento de escuelas de la Fundación Falconbridge, la gestión se concentra en la renovación y puesta a punto del espacio físico en el que funciona la institución escolar a la vez que se capacitan a profesores, coordinadores y estudiantes.

La gestión de contexto es complementaria a la gestión pedagógica y responde, igualmente, a la particularidad de las experiencias desarrolladas. En el caso de los ITAF y del Programa de Apadrinamiento de Escuelas, la gestión de contexto busca sensibilizar a la escuela frente a la realidad de las comunidades y convertirla en un factor de desarrollo de base. La escuela se vuelve así, una instancia clave para la organización de las comunidades, pues convoca a los actores comunitarios para movilizarse en torno a la educación de los niños, niñas y jóvenes.

La escuela opera, entonces, como un espacio público para la identificación de problemáticas educativas, y para la construcción y concertación de propuestas que buscan incidir en la realidad educativa de la comunidad. A diferencia del anterior tipo de experiencia, que moviliza a las organizaciones de base para incidir en la agenda de política pública, este tipo de experiencias moviliza a los actores comunitarios desde la escuela. En este sentido, la escuela se convierte en un espacio de organización para las comunidades desde el cual se busca la interacción con otras instancias sociales que pueden incidir, de manera conjunta, en la calidad de los aprendizajes de los niños, niñas y jóvenes. (Smurfitkappa, 2014; Falconbridge, 2005).

\section{Tercer tipo de experiencia: la apertura y cualificación de ambientes educativos en las comunidades}

En este tipo de trabajo se pueden ubicar las siguientes experiencias: Sistema de Aprendizaje Tutorial SAT, Fundación Wong (Ecuador) y Educación Interdimensional, Programa Crescer (Brasil).

Este tipo de experiencia se diferencia de los otros dos en que su trabajo se encuentra orientado hacia la construcción de ambientes sociales favorables para el desarrollo de los actores comunitarios, apunta a resolver 
necesidades educativas de los entornos comunitarios que respondan a las particularidades de los contextos. Por ello no operan simplemente como espacios complementarios a las instituciones formales de educación, sino que buscan suplir bien sea la ausencia de estas instituciones en los contextos, o la falta de pertinencia de sus propuestas para el desarrollo comunitario. En este sentido, las intervenciones se pueden dar en dos sentidos:

Uno de ellos es posibilitar nuevas interacciones entre los actores comunitarios mediadas por dispositivos pedagógicos que responden a las necesidades educativas del contexto. Es el caso del SAT, que a través de los Planes de Acción Comunitaria logra establecer conexiones entre los procesos productivos de las comunidades y los aprendizajes de los actores comunitarios. En este caso, el SAT configura una estrategia pedagógica alternativa a la escuela para potenciar el desarrollo de los actores comunitarios y favorecer su permanencia y arraigo en el contexto rural. (Wong, 2002)

El segundo es generar experiencias educativas innovadoras a través de organizaciones de base. Este es el caso del Programa CRESCER que favorece la creación de itinerarios educativos en las comunidades que se constituyen como un conjunto de oportunidades educativas que desencadenan experiencias de aprendizaje para los sujetos (Hedging Griffo, 2007).

Al igual que en el segundo tipo de intervención descrito anteriormente, las experiencias que se ubican en este tipo de intervención implementan un modelo pedagógico. Sin embargo, la diferencia aquí es que los modelos pedagógicos que se proponen no se pliegan a las finalidades de la educación escolarizada, sino que apuntan a la estructuración de experiencias educativas en espacios propiamente comunitarios.

El trabajo de las experiencias documentadas puede ubicarse así en una matriz teórica. Por una parte, los elementos estructurales de los campos de la educación y el desarrollo de base; por otra los problemas, que desde el punto de vista del actor social son problemas a trabajar, pero que desde el punto de vista del analista se convierten en posibilidad de explicación de la configuración del tejido social. La ubicación de cada experiencia se debe a su relación directa con uno o varios de los elementos estructurales así como la atención a una o varias de las problemáticas evidenciadas durante el estudio. 
Tabla 2. Matriz de ubicación de las experiencias.

\begin{tabular}{|c|c|c|c|c|c|c|c|}
\hline \multirow[b]{2}{*}{$\begin{array}{l}\text { Problemáticas } \\
\text { del territorio }\end{array}$} & & \multicolumn{3}{|c|}{ Desarrollo de base } & \multicolumn{3}{|c|}{ Educación } \\
\hline & & $\begin{array}{l}\text { Organización } \\
\text { de las } \\
\text { comunidades }\end{array}$ & $\begin{array}{l}\text { Capital } \\
\text { social }\end{array}$ & Participación & Saberes & Currículo & $\begin{array}{l}\text { Prácticas } \\
\text { pedagógicas }\end{array}$ \\
\hline \multirow{4}{*}{$\begin{array}{l}\text { Socialización } \\
\text { y aprendizajes } \\
\text { de los niños, } \\
\text { niñas y jovenes }\end{array}$} & $\begin{array}{l}\text { Formación de } \\
\text { subjetividades } \\
\text { políticas }\end{array}$ & & TPE & & $\begin{array}{l}\text { CR } \\
\text { SAT } \\
\text { FOCUS } \\
\text { ITAF }\end{array}$ & CR & $\mathrm{CR}$ \\
\hline & $\begin{array}{l}\text { Formación de } \\
\text { capital social }\end{array}$ & $\begin{array}{l}\text { OEC } \\
\text { FOCUS }\end{array}$ & $\begin{array}{l}\text { MEPE } \\
\text { OEC } \\
\text { TPE } \\
\text { FOCUS } \\
\text { JPE }\end{array}$ & $\begin{array}{l}\text { FOCUS } \\
\text { JPE }\end{array}$ & $\begin{array}{l}\text { SAT } \\
\text { FOCUS } \\
\text { TPE } \\
\text { SAT } \\
\text { ITAF }\end{array}$ & $\begin{array}{l}\text { SAT } \\
\text { ITAF }\end{array}$ & $\begin{array}{l}\text { TPE } \\
\text { SAT } \\
\text { ITAF }\end{array}$ \\
\hline & $\begin{array}{l}\text { Construcción } \\
\text { de ambientes } \\
\text { de aprendizaje }\end{array}$ & $\begin{array}{l}\text { OEC } \\
\text { ITAF }\end{array}$ & $\begin{array}{l}\text { TPE } \\
\text { JPE }\end{array}$ & TPE & $\begin{array}{l}\text { CR } \\
\text { SAT } \\
\text { FOCUS } \\
\text { ITAF } \\
\text { ADE }\end{array}$ & $\begin{array}{l}\text { TPE } \\
\text { CR } \\
\text { SAT } \\
\text { MEPE } \\
\text { ITAF } \\
\text { ADE }\end{array}$ & $\begin{array}{l}\text { CR } \\
\text { SAT } \\
\text { MEPE } \\
\text { FOCUS } \\
\text { ITAF } \\
\text { ADE }\end{array}$ \\
\hline & $\begin{array}{l}\text { Aprendizaje } \\
\text { de códigos } \\
\text { disciplinares }\end{array}$ & & & & $\begin{array}{l}\text { CR } \\
\text { SAT } \\
\text { ITAF } \\
\text { ADE }\end{array}$ & $\begin{array}{l}\text { TPE } \\
\text { CR } \\
\text { SAT } \\
\text { ITAF } \\
\text { ADE }\end{array}$ & $\begin{array}{l}\text { TPE } \\
\text { CR } \\
\text { SAT } \\
\text { ITAF } \\
\text { ADE }\end{array}$ \\
\hline
\end{tabular}

OEC: Oportunidades educativas y comunitarias. Fundación Arcor. JPE: Juntos por la educación. Fundaciones Arcor y Minetti. TPE: Todos por la educación. Instituto Camargo Correa. FOCUS: Fondo FOCUS. Fundación Corona. MEPE: Modelos escolares para la equidad. ITAF: Institutos técnicos agropecuarios y forestales. Fundación Smurfit. SAT: Sistemas de aprendizaje tutorial. Fundación Wong. CR: Programa Crescer. Instituto Hedging Griffo. ADE: Programa Apadrinamiento de escuelas. Fundación Falconbridge.

Nota. Recuperado de Desarrollo de base y educación. Informe Final por la Fundación Empresarios por la Educación (2008).

Hasta aquí se ha hecho una descripción de las experiencias de trabajo y se han identificado tres tipos de experiencias que se diferencian entre sí por su énfasis y estilo. Sin embargo, se han señalado al inicio del artículo, que los problemas del territorio son los que permiten la articulación entre desarrollo de base y educación. No se debe olvidar que el concepto de problema, en Luhmann (1998) es un criterio de referencia abstracto. Los problemas son por ello transversales y por lo mismo, generales, a las experiencias documentadas. 


\section{Problemáticas vinculantes}

\section{La formación de los sujetos}

Una lectura atenta de las experiencias permite percibir que todas apuntan a configurar procesos de construcción de subjetividad, tanto en los niños, niñas y jóvenes como en los actores del desarrollo de base, desde tres dimensiones. Una dimensión política, es decir, de construcción del sujeto como ciudadano participativo, una dimensión local, de reconocimiento de la pertenencia del sujeto al territorio, y una dimensión personal, es decir, de construcción del sujeto como identidad.

La formación de los sujetos, en este sentido, se realiza en dos ámbitos de socialización política que tienen lugar en las experiencias. En primer lugar, aparecen los procesos de socialización política de los niños, las niñas y los jóvenes, que se movilizan en una perspectiva enfocada a que los derechos de los niños tengan reconocimiento en las comunidades. Se trata pues, de la construcción de la infancia en el contexto de las comunidades, como una forma diferenciada de actuación social frente a los adultos, por lo tanto con posibilidades políticas específicas y con necesidades educativas que no sólo atañen a la institución escolar. Podría decirse que, de manera explícita o implícita, las experiencias conciben la educación como un factor crucial para la construcción de las identidades políticas de la infancia y la juventud. De esta forma, la educación no posee sólo una connotación de movilidad social para los sectores marginados, sino que se impulsa una mirada sobre lo educativo como espacio de construcción de identidades y de interacciones y permite a los niños, niñas y jóvenes comprender sus realidades e incidir en ellas de manera significativa.

Paralelo a esta perspectiva sobre la infancia como actor diferenciado en las comunidades, aparecen entonces, en las experiencias, componentes que apuntan a la construcción de la subjetividad política de los actores comunitarios a través de la conformación y fortalecimiento de las organizaciones de base y del establecimiento de puentes y puntos de conexión con otros actores sociales: con el Estado, con la empresa privada, con otras organizaciones.

\section{La formación de capital social}

El capital social supone la presencia de redes y estructuras sociales que se traducen en vínculos de solidaridad entre los sujetos, en normas, en 
un acumulado de experiencias y aprendizajes sociales que orientan la acción colectiva y en lazos y puentes que posibilitan el flujo de recursos y conocimientos entre diversas instancias sociales. En este sentido, el capital social aparece cuando las comunidades logran formalizar en cierto grado su capacidad de acción colectiva.

Una parte importante de las acciones que ponen en juego las entidades que promueven el desarrollo de base articulado a la educación tienen que ver con la constitución de una capacidad en las comunidades para comprender sus problemáticas en un contexto más amplio que aquel que, ligado al marginamiento, las interpreta como la simple presencia de carencias y deficiencias que deben ser suplidas por agentes externos a las comunidades. La formación del capital social se presenta, entonces, cuando las comunidades se organizan en torno a las dificultades o problemáticas educativas que presenta el contexto, para solucionarlas desde una perspectiva que implica la corresponsabilidad frente a los procesos educativos. Primero, como actores en las experiencias mismas y no sólo como sus beneficiarios, es decir, como agentes de desarrollo de las comunidades, que al apropiar los espacios de participación que se abren a través de los proyectos, decantan cada vez los aprendizajes y competencias que les permiten entrar en interlocución con otras instancias sociales. Segundo, como agentes que movilizan a otras instancias sociales y a otras organizaciones en función de construir soluciones a las problemáticas educativas que se presentan.

\section{La construcción de ambientes escolares y extraescolares que favorezcan los aprendizajes de los niños y niñas}

Esta problemática tiene que ver con la ausencia sentida en las comunidades de dispositivos de socialización que movilicen el desarrollo del territorio y que generen redes solidarias entre los niños, las niñas, los jóvenes, los adultos y las organizaciones sociales. Como tal, esta problemática se presenta en dos formas. Por un lado, las instituciones escolares, que representan para las comunidades el espacio educativo por excelencia, se restringen a una labor pedagógica centrada en los saberes escolares, pero sin consecuencias para la socialización de los niños y niñas desde perspectivas que los vinculen al territorio. Por otro lado, el territorio mismo no cuenta con espacios de socialización definidos por una acción pedagógica intencionada, es decir, inscrita en una propuesta educativa propia de las comunidades que permita 
tramitar las dificultades que se presentan en los contextos, tales como la drogadicción, el alcoholismo, el maltrato.

Frente a esto, las experiencias documentadas muestran que las intervenciones en el desarrollo de base y la educación no se pueden reducir a la implementación de acciones de refuerzo y apoyo a las prácticas pedagógicas escolares, sino que tienen que ver, en una medida importante, con la configuración de espacios para que los niños, las niñas y los jóvenes tengan acceso a la cultura y a la recreación, y puedan, a partir de ellos, construir formas de participación y de incidencia en el desarrollo de sus comunidades.

A partir de las experiencias documentadas se configura, así, una tendencia fuerte que liga las posibilidades de aprendizaje de los sujetos a la construcción de ambientes, antes que a la simple transmisión de conocimientos o a las capacitaciones. Así mismo, la construcción de los ambientes de aprendizaje postula a las organizaciones de base como agentes educativos que pueden dinamizar, a partir de sus propios saberes, experiencias de aprendizaje para otros actores comunitarios.

\section{El aprendizaje de los códigos o saberes escolares}

Ahora bien, la escuela en sí misma comporta una problemática para el desarrollo de base. No sólo por lo que se ha dado en llamar su actual crisis, sino sobre todo, por el potencial que representa para la construcción de tejido social, la organización de las comunidades y la socialización de los niños, niñas y jóvenes. Desde el centro de esa crisis y de esas posibilidades, el aprendizaje de los códigos o saberes escolares constituye una problemática para las comunidades, esto por tres razones primordiales: en primer lugar, los códigos escolares ponen en contacto a los niños, niñas y jóvenes con un acumulado de experiencias y conocimientos de la cultura que rebasan sus ámbitos inmediatos de experiencia y que les pueden permitir construir formas de comprensión claves para establecer relaciones entre sus realidades más próximas y las realidades más contextuales y globales. En segundo lugar, los aprendizajes de los saberes escolares se convierten en un factor importante de inclusión en las dinámicas productivas, económicas, sociales y culturales de la sociedad, por lo que la ausencia de estos aprendizajes se traduce, de hecho, en formas de exclusión de las comunidades. En tercer lugar, porque la significatividad del aprendizaje de los saberes escolares para los estudiantes se vuelve un factor que permite contrarrestar la deserción escolar. 
Por ello, las experiencias que se focalizan en la institución escolar tienen como uno de sus ejes principales de acción los procesos de formación del profesorado. La apertura y sensibilidad de la escuela hacia las problemáticas del contexto local conlleva, desde las experiencias, acciones tendientes a cualificar los procesos de gestión escolar a partir de la participación de los actores comunitarios en las decisiones que inciden en la calidad de los aprendizajes escolares.

\section{Conclusiones}

1. Existe un abanico amplio de iniciativas y acciones en las que el campo del desarrollo de base puede aportar al mejoramiento de la educación tales como la construcción de ambientes de aprendizaje, el mejoramiento de prácticas pedagógicas, la construcción de modelos educativos articulados al contexto, el fortalecimiento del capital social entorno al tratamiento de los problemas socioeducativos identificados y mayores niveles de participación de las organizaciones de base en torno a las problemáticas socioeducativas.

2. Es importante diferenciar entre impacto directo o indirecto al hablar de las incidencias que desde un campo se puede hacer sobre el otro. Así, las experiencias de TPE y FOCUS, aunque en diferente medida, inciden directamente en las prácticas pedagógicas de los docentes, pues trabajan con ellos en capacitación o en apropiación de nuevos materiales didácticos, mientras que experiencias como JPE y OEC, si bien no trabajan directamente aspectos estructurales del campo de la educación, indirectamente favorecen el mejoramiento de ellos.

3. Se puede concluir, a partir de la noción de equivalente funcional de Luhmann (1998), que todas las experiencias documentadas trabajan directa o indirectamente los aspectos concernientes al desarrollo de base y a la educación al situarse de cara a una problemática que los articula y que hace parte de las necesidades más sentidas de los territorios. El programa CRESCER, por ejemplo, que está centrado en trabajar la formación de sujetos desde el modelo de la educación interdimensional, al enfatizar en las competencias para la formación de ciudadanía, aporta a elevar las potencialidades que un territorio puede tener para conformar organizaciones de base, cualificar su capital social y aumentar sus niveles de participación en lo público. 
4. Al leer el cuadro en su conjunto, pueden apreciarse cuatro tendencias en el trabajo que desarrollan las experiencias documentas:

- Aquellas experiencias centradas en desarrollar un modelo pedagógico y que por lo mismo intervienen de manera directa en los tres elementos estructurales del campo de la educación (parte inferior derecha del cuadro), pero lo hacen vinculando a las organizaciones de base en alguna medida.

- El trabajo transversal en torno a los ambientes de aprendizaje. En esta tendencia se evidencia la preocupación, no sólo de los modelos pedagógicos sino de las organizaciones de base por ofrecerle a los niños, niñas y jóvenes adecuados ambientes de trabajo, incluso más allá de la escuela.

-El trabajo centrado en la formación y cualificación del capital social. Cinco experiencias de las nueve documentadas centran parte de sus esfuerzos en posicionar las problemáticas educativas como parte de las agendas de lo público y en elevar los niveles de interlocución al interior de la comunidad al proponer la elaboración de diagnósticos participativos y de planes de trabajo conjuntos. Al hacer esto, las problemáticas educativas se desescolarizan y pasan a ser asumidas como problemáticas del territorio.

- Cinco de las nueve experiencias se preocupan por el tema de los saberes que se trabajan en la educación de los niños y niñas. Esta tendencia demuestra la importancia que la pregunta por la pertinencia o no de los conocimientos que circulan en la escuela de cara a la formación de sujetos que puedan responder a las necesidades locales y de cara a poder estructurar currículos acordes con las dinámicas propias del desarrollo local.

5. No obstante, leído el cuadro en su conjunto se puede advertir una tendencia macro que consiste en poner el desarrollo de base al servicio de la educación más que en situar el campo de la educación en función del desarrollo de base. Esto no quiere decir, como ya se ha dicho, que los modelos pedagógicos alternativos que trabajan muchas de las experiencias documentadas no promuevan indirectamente el desarrollo de base, lo que se quiere hacer notar es la prioridad que tienen las problemáticas educativas sobre las problemáticas del desarrollo de base. 
6. Finalmente, en torno a los modelos pedagógicos se puede decir, que si bien el interés que mueve a un modelo pedagógico para aproximar las organizaciones de base a su trabajo está centrado en mejorar los saberes, en estructurar currículos más acordes con el contexto y en transformar las prácticas pedagógicas, no se debe desconocer el gran aporte que estos modelos pueden hacer a la cualificación del capital social y al mejoramiento de los niveles de participación de las organizaciones de base con las que trabajan.

\section{Referencias bibliográficas}

Arcor, F. (2006). Iniciativa Oportunidades Educativas Comunitarias. Documento de Trabajo.

Booth, A. J. (2000) Inclusion and exclusion policy in England: who controls the agenda? en F. Amstrong; D. Amstrong y L. Barton (eds): Inclusive Education. Policy, con texts and comparative perspectives, London, David Fulton Publishers.

CEPyD. (2001) Diagnóstico de la educación general básica en la ciudad de Córdoba. "Juntos por la Educación" Recuperado de http://www.cepyd.org.ar/admin/ upload/1378404801_diagnostico_educacion.pdf

Corona, F (2004). Focus. Un programa que aprendió de sí mismo. Bogotá, LitoCamargo Ltda.

Durston, J. (1995) La participación comunitaria en la gestión de la escuela rural. En CEPAL/OEA/Ediciones Sur. Educación, eficiencia y equidad. Santiago de Chile, LOM Ediciones Uda.

Empresarios por la Educación, F (2008). Desarrollo de base y educación. Informe final. Recuperado de http://fundacionexe.org.co/wp-content/uploads/2011/11/Estudiodesarrollo-de-base-y-educacion-Redeamerica.pdf

Empresarios por la Educación, F (2010). Modelos Escolares para la Equidad MEPE. Una ruta para aplicarlos. Recuperado de http://fundacionexe.org.co/wp-content/ uploads/2011/11/MEPE-Una-ruta-para-su-aplicaci\%C3\%B3n.pdf

Falconbridge, F (2005). Informe Anual 2005. Recuperado de http://www. fundacionfalconbridge.org/memorias/memoria2005.pdf

Fernández, J. V. M. (2009). La escuela centrada en la comunidad. Un modelo de escuela inclusiva para el siglo XXI. Revista Complutense de Educación, 20(1), 33.

García, P. C. (2005). Educación y diversidad. Málaga, Aljibe. 
Gajardo, M. y Puryear, J. (2003) Formas y reformas de la educación en América Latina. Santiago, PREAL.

Hedging Griffo, F (2007) Relatorio de actividades Instituto Hedging Griffo 2006/2007Recuperado de http://www.institutohg.org.br/siteihg/pdf/relatorio_IHG_2006-2007. pdf

Herrera, M. C. (2005). La construcción de cultura política en Colombia: proyectos hegemónicos y resistencias culturales. Bogotá, U. Pedagógica Nacional

Luhmann, N (1964) "Funktionale Methode und Systemtheorie", en Soziologische Aufklärung 1, pp. 31-53. Opladen, Westdeutscher.

Luhmann, N. (1998). Sistemas sociales: lineamientos para una teoría general (Vol. 15). Santafé de Bogotá, Anthropos Editorial.

Merino, J. V. (2005). Pedagogía social y educación social: reto de conocimiento y de acción para el siglo XXI, en J. Ruiz Berrio (Editor) Pedagogía y Educación ante el Siglo XXI, pp. 225- 251. Madrid, Universidad Complutense.

Merino, J.V. (2008) El movimiento de escuelas centradas en la comunidad. Cuadernos de Pensamiento, 21, 221-264.

Parrilla, A. (2002). Acerca del origen y sentido de la educación inclusiva. Revista de educación, (327), 11-29.

RedEAmérica (2007) Acompañamiento y cofinanciación a procesos organizativos para el desarrollo de base.

Torres, R. M. (2001). Participación ciudadana y educación. Una mirada amplia y 20 experiencias en América Latina. Documento encargado por la Unidad de Desarrollo Social y Educación (UDSE) de la OEA para su presentación en la Segunda Reunión de Ministros de Educación del Consejo Interamericano para el Desarrollo Integral. Recuperado de: http://www.unesco.org/education/efa/partnership/oea_document.pdf

Smurfitkappa, F (2014). Informe Anual 2014. Recuperado de http://www.smurfitkappa. $\mathrm{com} / \mathrm{vHome} / \mathrm{co} /$ Sustainability/Social/Communitylnvolvement/Documents/FSKC\%20 Informe2014\%20final.pdf

Trilla, J. B. (1995). La escuela y el medio: Una reconsideración sobre el contorno de la institución escolar. En Volver a pensar la educación (Congreso Internacional de Didáctica) (pp. 217-231). Fundación Paideia.

Trilla J B. (1999). La ciudad educadora. De las retóricas a los proyectos. Cuadernos de pedagogía, (278), 44-50. 
UNESCO (2013). Situación Educativa de América Latina y el Caribe: Hacia la educación de calidad para todos al 2015. Recuperado de http://www.unesco.org/new/fileadmin/ MULTIMEDIA/FIELD/Santiago/images/SITIED-espanol.pdf

Wong, F (2002) Educación. Recuperado de http://www.fundacionwong.org/educacion_ rural.php 